\title{
EL ORIGEN DE LA CLÁUSULA DE LA MAYOR PROTECCIÓN DE LOS DERECHOS HUMANOS*
}

\author{
The origin of the Human Rights greater protection clause
}

\author{
Pablo Sánchez-Molina \\ Investigador Predoctoral (Becas de la Obra Social «la Caixa») en Derecho Constitucional \\ Universidad de Sevilla \\ psanchez12@us.es
}

doi: http://dx.doi.org/10.18543/ed-66(1)-2018pp375-391

Recibido: 12.04 .2018

Aceptado: 26.06.2018

\section{Resumen}

La cláusula de la mayor protección en los tratados sobre Derechos Humanos se configuró con el objetivo de conciliar la ampliación de los Estados parte de la Organización Internacional del Trabajo y el mantenimiento del nivel de protección de los derechos en los Estados industrializados. En el presente trabajo profundizamos en el origen y naturaleza de esta figura esencial para la construcción de un sistema multinivel de protección de los Derechos Humanos.

* Cómo citar / Citation 'Chicago-Deusto' (Autor-fecha / Author-date / Lista de referencias / Reference list entries): Sánchez-Molina, Pablo. 2018. «El origen de la cláusula de la mayor protección de los Derechos Humanos». Estudios de Deusto 66, n. ${ }^{\circ} 1: 375-391$. doi: http://dx.doi.org/10.18543/ed-66(1)-2018pp375-391.

Agradezco al profesor Ángel Rodríguez sus valiosas aportaciones para la realización de este trabajo. Asimismo, al personal de biblioteca de la Facultad de Derecho de la Universidad de California, Berkeley, en especial a Marci Hoffman (associate director). Esta estancia de investigación fue posible gracias a la financiación del plan propio de la Universidad de Sevilla y del proyecto de investigación «Reforma electoral y derechos de participación de los extranjeros residentes en España: el derecho de sufragio en las elecciones generales, europeas, autonómicas y locales (Votex4e)». Ministerio de Economía y Competitividad (referencia DER2012-3441). 


\section{Palabras clave}

Origen; cláusula; mayor protección; DDHH; OIT; estándar de protección.

\section{Abstract}

The more favourable protection clause on Human Rights Treaties was configured to achieve this aim: reconcile the International Labour Organization enlargement and the maintenance of the level of Rights protection in the industrialized States. In this article we deepen in the origin and nature of this essential figure for the construction of a Human Rights multilevel system.

\section{Keywords}

Origin; clause; more favourable; HHRR; ILO; standard of protection. 


\begin{abstract}
SUMARIO: I. INTRODUCCIÓN. II. LA PROHIBICIÓN DE LIMITACIONES EXTRÍNSECAS Y LA CLÁUSULA DE LA MAYOR PROTECCIÓN DE LOS DERECHOS HUMANOS. III. EL ORIGEN DE LA CLÁUSULA DE LA MAYOR PROTECCIÓN DE LOS DERECHOS HUMANOS A TRAVÉS DE UNA REFORMA DE LA CONSTITUCiÓN DE LA ORganización INTERnACIONAL DEL TRABAJO (OIT). IV. CONCLUSIONES. BibLIOGRAFÍA.
\end{abstract}

\title{
I. INTRODUCCIÓN
}

El Derecho Constitucional constituye el terreno sobre el que se construyen las sociedades contemporáneas y, dentro de este, los Derechos Humanos (en adelante, DDHH) conforman una de sus partes más imprescindibles, sin ellos no se podrían alcanzar las condiciones mínimas para que la construcción social sea viable a largo plazo. Como no puede ser de otro modo, el derecho evoluciona conforme lo hace la sociedad aunque, normalmente, con algo de retraso. La evolución del ordenamiento, bien vía interpretativa o de reforma, es, con carácter habitual, positiva debido a que a su través se facilita el acercamiento de una regulación a las circunstancias actuales. Es por ello que en la evolución de los Derechos Humanos entran en juego una gran número de factores que superan el ámbito del derecho. Un buen ejemplo lo constituye la «aculturación» ${ }^{1}$.

En esta evolución la labor de las fuentes del derecho es esencial. Estas constituyen la vía de acceso de las posibles modificaciones en el ámbito de los DDHH a derecho aplicable. Nos encontramos, por lo tanto, ante unas fuentes que han ido incorporando la noción de globalización en el ámbito jurídico, superando el carácter nacional que tradicionalmente había impe$\mathrm{rado}^{2}$. En la actualidad, el derecho de producción interna interacciona con otra serie de normas, de producción externa, que han revolucionado el mundo del derecho y, por ello, la evolución en el ámbito de los DDHH cuenta con una doble perspectiva: nacional e internacional. Esta doble perspectiva, en ocasiones, produce el mismo efecto al constituirse como dos afluentes que desembocan en el río de los derechos nacionales y, presidida por el diálogo y la recepción mutua, ha llevado a la incorporación en las constituciones nacionales de mecanismos de interpretación y recepción del derecho internacional de los DDHH.

El ámbito de la protección internacional de los DDHH es considerablemente homogéneo. Al analizar los distintos tratados internacionales que

1 Ver GoOdMan y JinKs 2004: 638 y ss.

${ }^{2}$ Una visión tradicional del derecho internacional puede verse, por ejemplo, en CABALEIRO 1962: 105 y ss. 
recogen los acuerdos mínimos en este ámbito no encontramos grandes diferencias en lo que a derechos reconocidos se refiere. Esta homogeneidad no implica una total identidad, por lo que los diferentes instrumentos están capacitados para otorgar diversos estándares de protección a un determinado derecho. Aun así, los instrumentos internacionales de protección de los DDHH no son la única vía a través de la que se configuran estos derechos esenciales, sino que la protección efectiva derivará, sobre todo, de las regulaciones de los Estados. Los preceptos reconocidos en el derecho internacional y nacional coinciden, normalmente, en su tenor y extensión pero, en ocasiones, las diferencias existen.

Con carácter general, los Convenios de protección de los Derechos Humanos otorgan una protección mínima que todos los Estados parte tienen que alcanzar y pueden superar. En este sentido, el principio de subsidiariedad, del que deriva la condición de los tratados internacionales de protección de los Derechos Humanos como un mínimo común de protección (lo que no obsta para que los Estados otorguen un nivel de protección mayor) constituye un concepto a tener muy en cuenta. La razón radica en la diversidad de Estados signatarios y en la necesidad de fijar unas bases comunes a todos ellos. La consideración de los Tratados Internacionales de protección de los DDHH como mínimo común ha llevado a que la mayoría de los sistemas de protección internacional de los DDHH fijen cláusulas para impedir que esta característica suponga una traba en la evolución de estos derechos. En este punto, la cláusula de la mayor protección de los DDHH se nos presenta como esencial en la correcta evolución de estos derechos imprescindibles en cualquier sociedad democrática. En este artículo, nos centraremos en analizar el origen de la cláusula de la mayor protección de los DDHH. Aún así, antes de ello, en el siguiente apartado, nos referiremos a la regulación contenida en la Convención de Viena sobre derecho de los tratados así como el desarrollo de aquellos criterios contenidos en los tratados internacionales de protección de los DDHH y que limitan su propia aplicación.

\section{LA PROHIBICIÓN DE LIMITACIONES EXTRÍNSECAS Y LAS CLÁUSULAS DE LA MAYOR PROTECCIÓN DE LOS DERECHOS HUMANOS}

La proliferación de tratados internacionales sobre DDHH contribuyó al incremento de su peso específico como fuente del derecho dentro del sistema multinivel de protección ${ }^{3}$. Este incremento, que no solo afectó al ámbito de

${ }^{3}$ Reconociendo la importancia cada vez mayor de los tratados como fuente del derecho internacional y como medio de desarrollar la cooperación pacífica entre las naciones, 
los DDHH sino a muchos otras materias, hizo necesario la configuración de una normativa que posibilitase la solución de posibles conflictos existentes. La Convención de Viena sobre derecho de los tratados recoge, a lo largo de su articulado, las normas aplicables para una correcta coexistencia de estas normativas internacionales ${ }^{4}$. Para ello, los propios Estados, a través de esta Convención, se han otorgado unas reglas de juego. Entre ellas encontramos un criterio aplicativo de tratados que contienen la misma materia, recogido en el artículo $30^{5}$. En este precepto se establecen tres reglas que podemos dividir en dos grupos: cuando el tratado indica expresamente la

sean cuales fueren sus regímenes constitucionales y sociales. Segundo considerando de la Convención de Viena sobre el Derecho de los Tratados. U.N. Doc A/CONF.39/27 (1969), 1155 U.N.T.S. 331 (adoptada en Viena el 23 de mayo de 1969 y en vigor desde el 27 de enero de 1980).

${ }^{4}$ Art. 1 de la Convención de Viena relativo al «alcance de la presente Convención»: «La presente Convención se aplica a los tratados entre Estados».

5 Art. 30 de la Convención de Viena sobre el derecho de los tratados (Aplicación de tratados sucesivos concernientes a la misma materia):

«1. Sin perjuicio de lo dispuesto en el articulo 103 de la Carta de las Naciones Unidas, los derechos y las obligaciones de los Estados partes en tratados sucesivos concernientes a la misma materia se determinaran conforme a los párrafos siguientes. 2. Cuando un tratado especifique que está subordinado a un tratado anterior o posterior o que no debe ser considerado incompatible con ese otro tratado prevalecerán las disposiciones de este último. 3. Cuando todas las partes en el tratado anterior sean también partes en el tratado posterior, pero el tratado anterior no quede terminado ni su aplicación suspendida conforme al articulo 59, el tratado anterior se aplicara únicamente en la medida en que sus disposiciones sean compatibles con las del tratado posterior. 4. Cuando las partes en el tratado anterior no sean todas ellas partes en el tratado posterior: a) en las relaciones entre los Estados partes en ambos tratados se aplicará la norma enunciada en el párrafo 3. b) en las relaciones entre un Estado que sea parte en ambos tratados y un Estado que sólo lo sea en uno de ellos, los derechos y obligaciones recíprocos se regirán por el tratado en el que los dos Estados sean partes. 5. El párrafo 4 se aplicará sin perjuicio de lo dispuesto en el articulo 41 y no prejuzgará ninguna cuestión de terminación o suspensión de la aplicación de un tratado conforme al artículo 60 ni ninguna cuestión de responsabilidad en que pueda incurrir un Estado por la celebración o aplicación de un tratado cuyas disposiciones sean incompatibles con las obligaciones contraídas con respecto a otro Estado en virtud de otro tratado».

U.N. Doc A/CONF.39/27 (1969), 1155 U.N.T.S. 331. Viena, 23 de mayo de 1969 (entrada en vigor el 27 de enero de 1980). Pueden verse algunos comentarios sobre la Convención de Viena sobre el derecho de los tratados en CoRTEn, Oliver y KLEIN, Pier [ed.] (2011): The Vienna Conventions on the Law of Treaties. A commentary, Oxford University Press. Págs. 764 y ss; Villinger, Mark E. (2009): Commentary on the 1969 Vienna Convention on the Law of the Treaties, Martin Nijhoff Publishers, Leiden/Boston o Vanneste, Féderic (2010): General International Law before Human Rights Courts. 
subordinación a otro (apartado segundo) y cuando esta remisión no se produce (apartado tercero y cuarto).

Dentro de la primera categoría, la Convención se refiere a aquellos tratados que indican la «subordinación» o «compatibilidad» con otros. Ello implica la prevalencia de unos tratados sobre otros porque así lo indica uno de ellos en el propio instrumento internacional. Así, siempre que en un tratado se indique una regla aplicativa en favor de otro se aplicará la disposiciones de este último ${ }^{6}$.

Dentro de la segunda encontramos aquellos casos en los que no se indica ninguna prevalencia en el propio tratado. Y, a su vez, dos reglas diferenciadas dentro de esta categoría. Una para aquellos supuestos en los que dos tratados sean sucesivos o, al menos, cuando nos encontremos ante dos Estados que hayan ratificado ambos. En este supuesto se aplicará el tratado posterior excepto en aquello en lo que el anterior sea compatible y siempre que este no haya quedado «eliminado» o «terminado» ${ }^{7}$. Y otra, en los supuestos en los que mientras que un Estado es parte de ambos tratados, el anterior y el posterior, y el otro sea parte de solo uno de ellos. En su caso, se aplicaría aquél del que ambos sean parte ${ }^{8}$.

De este breve comentario sobre el criterio aplicativo de la Convención de Viena nos interesa ahora el problema de la existencia de criterios similares en el texto de los tratados y preguntarnos acerca de cual debe prevalecer sobre el criterio general de aplicación del tratado posterior sobre el anterior. En efecto, los tratados internacionales de DDHH cuentan, entre su articulado, con algunos criterios que limitan la aplicación del propio instrumento internacional al que dan vida. Estos criterios limitativos tratan de imposibilitar usos de sus preceptos que vayan en contra del espíritu del tratado. Dentro de este ámbito encontraríamos dos tipos de criterios limitativos, intrínsecos y extrínsecos.

En primer lugar, los preceptos que establecen prohibiciones de limitaciones intrínsecas hacen referencia a la imposibilidad de aplicar un instrumento internacional de protección de DDHH con la finalidad de limitar o suprimir los derechos reconocidos en el mismo. La prohibición, por tanto, del abuso de derecho se nos presenta como una prohibición de aplicación o interpretación de un determinado tratado internacional que traiga como consecuencia un menoscabo del propio tratado. Así, artículos como el 30 DUDH, 17

Assessing the Specialty Claims of International Human Rights Law, Intersentia, AntwerpOxford-Portland. Págs. 213 y ss.

${ }^{6}$ Art. 30.2 de la Convención de Viena sobre el derecho de los tratados.

7 Art. 30.3 y 30.4.a de la Convención de Viena sobre derechos de los tratados.

8 Art. 30.4.b de la Convención de Viena sobre derechos de los tratados. 
CEDH, 54 CDFUE o 5.1 PIDCP, entre otros, contienen este tipo de prohibiciones ${ }^{9}$.

En segundo lugar, la prohibición de limitaciones extrínsecas hace referencia a la imposibilidad de que la aplicación o interpretación de un derecho reconocido en un Tratado Internacional de protección de los DDHH conlleve la limitación de otro externo que le sea igualmente aplicable a un Estado parte.

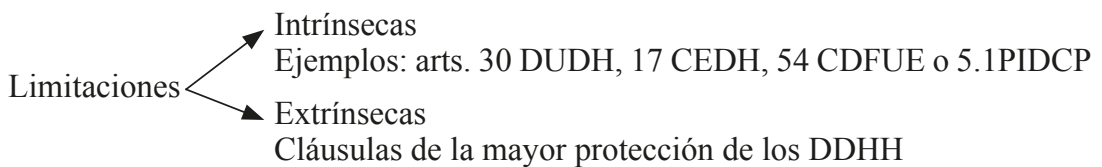

Dentro de la prohibición de limitaciones extrínsecas, como hemos indicado, encontramos las cláusulas de la mayor protección de los Derechos Humanos. Una primera conceptualización de este principio radicaría en la imposibilidad de que los Estados parte de un tratado internacional de protección de los Derechos Humanos utilizasen las previsiones recogidas en el propio tratado con la finalidad de limitar la protección nacional, esto es, que se

9 Art. 30 DUDH: «Nada en esta Declaración podrá interpretarse en el sentido de que confiere derecho alguno al Estado, a un grupo o a una persona, para emprender y desarrollar actividades o realizar actos tendientes a la supresión de cualquiera de los derechos y libertades proclamados en esta Declaración».

Art. 17 CEDH: «Ninguna de las disposiciones del presente Convenio podrá ser interpretada en el sentido de implicar para un Estado, grupo o individuo, un derecho cualquiera a dedicarse a una actividad o a realizar un acto tendente a la destrucción de los derechos o libertades reconocidos en el presente Convenio o a limitaciones más amplias de estos derechos o libertades que las previstas en el mismo».

Art. 54 CDFUE: «Ninguna de las disposiciones de la presente Carta podrá ser interpretada en el sentido de que implique un derecho cualquiera a dedicarse a una actividad o a realizar un acto tendente a la destrucción de los derechos o libertades reconocidos en la presente Carta o a limitaciones más amplias de estos derechos y libertades que las previstas en la presente Carta».

Art. 5.1 PIDCP y art. 5.1 PIDESC: «Ninguna disposición del presente Pacto podrá ser interpretada en el sentido de conceder derecho alguno a un Estado, grupo o individuo para emprender actividades o realizar actos encaminados a la destrucción de cualquiera de los derechos y libertades reconocidos en el Pacto o a su limitación en mayor medida que la prevista en él».

Art. 29.a CADH: «Ninguna disposición de la presente Convención puede ser interpretada en el sentido de: a. permitir a alguno de los Estados partes, grupo o persona, suprimir el goce y ejercicio de los derechos y libertades reconocidos en la Convención o limitarlos en mayor medida que la prevista en ella». 
produzca una regresión en el ámbito de protección. En este sentido, la Asamblea General de la Organización de Estados Americanos (OEA) ha definido «medidas regresivas» como «aquellas disposiciones o políticas cuya aplicación signifique un retroceso en el nivel del goce o ejercicio de un derecho protegido $»^{10}$. Alguna doctrina ha conceptualizado la regresividad como un límite a la actuación de los poderes públicos y, a su vez, como una garantía de protección de los derechos de los individuos ${ }^{11}$.

Podríamos preguntarnos si para aplicar siempre aquella perspectiva más favorable en pro de los Derechos Humanos es necesaria la existencia de una cláusula a nivel internacional o, por el contrario, es suficiente con limitaciones y garantías en el ámbito estatal o, incluso, podríamos considerar que esta situación deviene en un principio interpretativo de carácter general y cuyo argumento fundamental deriva de un hecho en concreto: la pluralidad de fuentes internas. Para dar respuesta a estas cuestiones es necesario conocer el ámbito de la mayor protección de los DDHH en toda su extensión partiendo de todos los sujetos implicados: los sistemas internacionales de protección de los DDHH y los propios Estados parte de dichos tratados.

Desde un punto de vista internacional, a primera vista, el motivo de la inclusión de estas cláusulas radicaría en condicionar la aplicabilidad de las normas. Por ello, un tratado que recoja en su texto la cláusula de la mayor protección estará limitando la propia aplicación de su normativa en los supuestos que exista otra norma más protectora ${ }^{12}$. Estas cláusulas, también denominadas «saving clause» ${ }^{13}$, buscan en última instancia que no se vaya en contra de la esencia de la salvaguarda de los Derechos Humanos desde una perspectiva dinámica y evolutiva que iría más allá de la permisividad a la hora de aplicar normas más protectoras. Si a través de estas cláusulas los Estados parte, o incluso otros tribunales internacionales, gozaran de la posibilidad de elegir entre la aplicación de la normativa internacional o la suya propia esta perdería virtualidad y eficacia para una mayor protección. En esta línea, los Estados estarían legitimados tanto para proteger como limitar los DDHH en el caso que vieran oportuno. Es por ello que para conocer la tendencia de esta cláusula no solo basta con analizar el tenor literal de los preceptos que la contienen sino estudiar, en última instancia, su aplicación práctica.

El carácter extrínseco de este fenómeno lo diferencia de aquellas otras disposiciones que prohíben una limitación intrínseca y que no serán

10 AG/RES. 2074 (XXXV-O/05). Normas para la confección de los informes periódicos previstos en el Protocolo de San Salvador. A través de TORREs ZúÑIgA 2015: 96.

11 TORRES ZÚÑIGA 2015: 98.

12 RACHOVITSA 2016: 77.

13 DETRICK 1999: 712. 
analizadas en el presente artículo. Por ello, tras haber enmarcado nuestro objeto de estudio dentro de las prohibiciones de limitación extrínsecas, debemos probar si nos encontramos ante una cláusula aplicativa, interpretativa o bifronte (incluyendo a ambas). La doctrina analizada se ha inclinado, a través de diferentes autores, por las dos primeras posibilidades, lo que nos llevaría a tener en cuenta la tercera vía, de carácter ecléctico o, por el contrario, por la mutabilidad de la cláusula, bien como elemento interpretativo, aplicativo $\mathrm{o}$, como ninguna de los dos, dependiendo del instrumento internacional de protección de los DDHH ante el que nos encontremos.

Por un lado, podríamos entender que conectando la cláusula de la mayor protección con el art. 30 de la Convención de Viena, las previsiones de limitaciones extrínsecas serían aplicativas y complementarias a estas encuadrándose, en nuestra opinión, en el segundo apartado del citado precepto ${ }^{14}$. Por otro, este tipo de cláusulas podría englobarse dentro de las interpretativas y, por tanto, complementaria a los arts. 31-33 de la Convención de Viena ${ }^{15}$.

No nos encontramos en disposición de enmarcar las cláusulas que prevén la prohibición de limitaciones extrínsecas como una regla interpretativa o aplicativa. La principal diferencia entre ambas radicaría en que mientras la técnica interpretativa impediría una lectura del precepto internacional en el sentido de restringir la normativa aplicable a un determinado Estado, la vertiente aplicativa impediría el uso, digamos, completo de aquella normativa internacional menos favorable para aplicar, en exclusiva, el estándar nacional de protección de los derechos. Por último, esta diferenciación podría responder a una construcción artificial donde aplicación e interpretación actuarían como dos caras de una misma moneda dependiendo del sistema internacional de los DDHH en el que se encuadre ${ }^{16}$. A estos, y otros, interrogantes intentaremos dar respuesta en el transcurso del presente capítulo.

${ }^{14}$ En este sentido se muestra Alkema: «Article 60 is rather to be considered as a rule for the application of treaties itself, complementary to notably Article 30 VCLT entitled "[a]pplication of successive treaties relating to the same subject-matter». In contrast to Article $30 \mathrm{VCLT}$, Article $60 \mathrm{ECHR}$ is restricted neither to the 'same subject-matter' nor to 'successive treaties'». Alkema, Evert. A.: «The enigmatic no-pretext clause: Article 60 of the European Convention on Human Rights» en KLABBERS y LEFEBER 1998.

15 Así, Vanneste en el capítulo títulado «Special approach towards interpretation of Human Rights treaties (31-33 VCLT)»: «It is often said that human rights courts have developed "special" rules of interpretation [...] There is no specific rule in the ECHR, except for Article 53 safeguarding the existing human rights. 722 And Article 29 ACHR does not clarify the distinction between creation and recognition of human rights». VANNESTE 2010: 213-214.

${ }^{16}$ «The preparatory work and the text of the clauses do not offer conclusive evidence as far as their precise nature and function are concerned. The provisions of the ACHR and the ECHR appear to adopt a particular terminology, which relates mainly to the interpre- 
Es por ello que nos encontramos ante una disposición normativa vinculante para los Estados pero sometida a condiciones para su ejercicio dependiendo del tratado internacional ante el que nos encontremos ${ }^{17}$.

Tras habernos referido a la naturaleza de las cláusulas limitativas en los tratados internacionales de protección de los $\mathrm{DDHH}$, abordaremos el origen y razón de ser de la cláusula de la mayor protección de los DDHH.

\section{EL ORIGEN DE LA CLÁUSULA DE LA MAYOR PROTECCIÓN DE LOS DERECHOS HUMANOS A TRAVÉS DE UNA REFORMA DE LA CONSTITUCIÓN DE LA ORGANIZACIÓN INTERNACIONAL DEL TRABAJO (OIT)}

Al contrario de lo que pudiera parecer a primera vista, sobre todo a raíz de la lectura de la introducción de este trabajo, el primer antecedente de cláusula de mayor protección de los Derechos Humanos no lo encontramos en el Convenio Europeo de Derechos Humanos sino en una de las reformas de la Organización Internacional del Trabajo (OIT $)^{18}$. De hecho, en los motivos de su inclusión se encuentra una de las claves de la presente investigación que deriva de la noción de fragmentación del derecho. En este sentido, el carácter internacional de estos instrumentos de protección de los Derechos Humanos lleva a que, tras la consolidación primera en un grupo reducido de Estados, se plantee una ampliación de los sujetos implicados. Así, a la Constitución de la OIT le siguieron otras disposiciones de la mayoría de tratados internacionales de protección de los $\mathrm{DDHH}^{19}$.

tation task, whereas the wording of the ICCPR, ICESCR and CRC leans towards the application task», RACHOVITSA 2016: 82. Ver también FOROWICZ 2010: 26 y ss.

17 «First, it must be shown that a more favourable provision under an international treaty is binding on the respondent State». RACHOVITSA 2016: 99.

18 «This affects particulary the conventions of the International Labour Organisation (ILO) in which the clause is frequently included. It contains a most favoured protection clause [...]». AlKema 1998: 53.

19 Convenio Europeo de Protección de los Derechos Humanos (1950), Convención sobre el Reconocimiento y la Ejecución de las Sentencias Arbitrales Extranjeras (1958), Carta Social Europea (1961), Convención internacional sobre la eliminación de todas las formas de discriminación racial (1965), Pacto Internacional de Derechos Civiles y Políticos (1966), Pacto Internacional de Derechos Económicos, Sociales y Culturales (1966), Convención Americana de Derechos Humanos (1969), Convención sobre la eliminación de todas formas de discriminación contra la mujer (1979), Convenio sobre Prohibiciones o Restricciones en el Empleo de Ciertas Armas Convencionales que Pueden Considerarse Excesivamente Nocivas o de Efectos Indiscriminados (1980), Convención contra la tortura y otros tratos o penas crueles, inhumanos o degradantes (1984), Convención de Derechos del Niño (1989), Convención internacional sobre la protección de los derechos de todos los trabajadores migratorios y sus familias (1990), Convención sobre los derechos 
Cuatro semanas antes del Desembarco de Normandía, como se sabe, hecho histórico que fue decisivo para la victoria aliada en la Segunda Guerra Mundial, se reunieron en Filadelfia los representantes de los 41 Estados miembros de la OIT (organización universal constituida a partir del Tratado de Versalles ${ }^{20}$. Esta fue una de las dos convenciones que organizó la OIT durante el transcurso de la contienda y cuyo resultado fue la Declaración de Filadelfia que introdujo una reforma de la versión original de la Constitución de la OIT, de $1919^{21}$. A través de esta reforma se añadió al texto original una serie de disposiciones, entre las que se encontraba una cláusula ideada para dar respuesta a la problemática planteada ante la expansión de la OIT al resto de continentes ${ }^{22}$.

Los instrumentos internacionales de protección de los Derechos Humanos tienen, a nuestro entender, dos objetivos principales. El primero de ellos deriva de su carácter internacional mientras que el segundo radica en su eficacia. Dentro de su ámbito de actuación, bien regional o universal, los tratados internacionales de protección de DDHH tratan de sumar en su seno a la mayoría de Estados. Esta vocación incluyente se convierte en una realidad práctica en el momento en el que la mayor parte de los Estados firman y ratifican el tratado, pero sus objetivos en pro de la protección de los derechos no tendrían ninguna relevancia si sus resoluciones no fueran respetadas. De otro modo nos encontraríamos ante un compendio de buenas intenciones sin ninguna transcendencia jurídica. Para salvar este dualismo, alcanzar una protección efectiva que, a su vez, incluya a un número significativo de Estados parte, los tratados internacionales, por lo general, se basan en el principio de subsidiariedad.

Ante esta dicotomía se encontró la Organización Internacional del Trabajo una vez acabada la Segunda Guerra Mundial: ampliar los Estados parte sin limitar la protección y el grado de cumplimiento. No solo bastaba con una limitación de los derechos consagrados en la ILO original para que la ampliación tuviese éxito sino que se tendría que evitar, a su vez, que la protección disminuyese en aquellos Estados industrializados. Mientras una disminución de los estándares podría «afectar al prestigio de la función normativa y, por lo tanto, alienar a los países industrializados», mantener los estándares de institución podría dejar «prácticamente sin sentido» el compromiso de aquellos Estados menos desarrollados que, materialmente, no pudieran alcanzar

de las personas con discapacidad (2006), Carta de Derechos Fundamentales de la Unión Europea (2000) y Convención internacional para la protección de todas las personas contra las desapariciones forzadas (2010), entre otros.

20 Del 20 de abril al 12 de mayo de 1944. Chebali 1989.

21 Junto con la Convención de Nueva York de 1941. Chebali, 1989: 19.

22 Murray 2001: 62 y Chebali 1989: 19. 
el nivel de protección fijado ${ }^{23}$. El artículo 19.8 de la Constitución de la OIT pretendía conjugar esta dicotomía sin afectar a la protección nacional de los derechos, algo aparentemente contradictorio, con la siguiente previsión: «En ningún caso podrá considerarse que la adopción de un convenio o de una recomendación por la Conferencia, o la ratificación de un convenio por cualquier Miembro, menoscabará cualquier ley, sentencia, costumbre o acuerdo que garantice a los trabajadores condiciones más favorables que las que figuren en el convenio o en la recomendación».

En relación con la naturaleza de la OIT, a diferencia de otros tratados, el uso de la cláusula de mayor protección no puede verse limitado por la existencia de conflictos de derechos pues, como aparece en el texto del precepto, se refiere a aquellas condiciones más favorables para los trabajadores. Su causa deviene de la naturaleza sectorial de la OIT favorable a extensiones favor-libertatis. Sin embargo, su incorporación en otros tratados internacionales de protección de los DDHH que incluyen un ámbito más amplio puede provocar que una mayor protección de un derecho conlleve la limitación de otro. En estos supuestos, la cláusula contará con una problemática adicional y requerirá de una delimitación teórica muy precisa para que los órganos encargados de fiscalizarla puedan aplicarla o rechazarla.

La cláusula del art. 19.8 sirvió como contrapeso a la limitación de algunos principios fundacionales tras la reforma de la Constitución de la OIT conjugando un mínimo de protección internacional, que permitiese cumplir a los nuevos Estados parte, con otro estándar más elevado en aquellos Estados más consolidados $^{24}$. Y, además, su respeto se configuró como la «piedra angular»

${ }^{23}$ «But after the Second World War the heterogeneity of the member States and the proliferation of regional organisations raised a dilemma: should the Organisation consider lowering its standards so as to bring them within the reach of newly independent countries, or should it strive to maintain them at their traditional level? Either way, there was a risk. A lowering of standards could affect the prestige of the standard setting function and thus alienate the industrialised countries. If, on the other hand, the level of standards was maintained, in some cases at least the undertaking made by States to apply them might become largely meaningless; in particular, ratifications by newly independent States might well, in most cases, have a merely token value. In other words, the risk was either that a "regionalised" pattern of ratifications might develop, depending on the higher or lower level of the standards concerned, or that the number of member States genuinely able to comply with the legal obligations the standards entailed and to base their social policy on them would be limited». CHEBALI 1989: 205.

${ }_{24}$ «In fact, there were a number of tensions between the Declaration of Philadelphia and the rest of the ILO's Constitution and the regulatory system it established: some of Declaration's provisions implied limitations to the original ILO founding principles, while others altered the scope of ILO interests. The extension of scope to 'all human beings' implied that it was not just those who were capable of or willing to disrupt social stability who should receive the ILO's protection». MURRAY 2001: 64. 
en la búsqueda de un equilibrio, en los Estados, entre las reivindicaciones laborales de los trabajadores, por un lado, y las necesidades de la industria, por otro, que trató de impedir que la legislación laboral constituyese un freno al crecimiento sin que se vieran perjudicadas las condiciones laborales de los trabajadores. A esta utopía, así lo ha denominado una parte de la doctrina, se le intentó dar solución estableciendo un mínimo común de protección de los derechos laborales sobre el que los Estados construirían la protección interna ${ }^{25}$.

El establecimiento de este mínimo común de protección conllevaba un riesgo, y es aquí donde la cláusula contenida en el art. 19.8 de la Constitución de la OIT adquiere la condición de «piedra angular». La expresión «the raceto-the-bottom» hace referencia a este fenómeno que supone una tendencia a la estandarización de los derechos laborales hasta el mínimo exigido y que respondería a una idea establecida en uno de los considerandos de la Convención de Filadelfia: «poverty anywhere constitutes a danger to prosperity everywhere $»^{26}$. En el ámbito laboral, como en otros, las mejoras de las condiciones de los trabajadores suponen un gasto para los empleadores o para el Estado. Es una relación directamente proporcional, por lo que la progresiva conquista de derechos laborales supondría un incremento de las partidas económicas destinadas a sufragarlas. Es por ello que en un campo, en muchos casos, extremadamente competitivo, el respeto de unas condiciones laborales «realmente humanas» por la gran mayoría de países del planeta tendría un efecto multiplicador para la progresiva realización de los derechos de los trabajadores. Como es sabido, la competitividad del mercado laboral tiene unos efectos decisivos en la economía nacional por lo que el respeto por los Estados de unos derechos mínimos supondría que la competitividad entre los propios Estados se igualase mientras que lo contrario supondría, como establece uno de los considerandos de la Constitución de la OIT, «un obstáculo a los esfuerzos de otras naciones que deseen mejorar la suerte de los trabajadores en sus propios países».

La expansión de la OIT, planteada en la Convención de Filadelfia ${ }^{27}$, se materializó en las décadas posteriores al duplicarse el número de

${ }^{25}$ La decisión de disminuir el nivel de protección en ciertos ámbitos no fue unánime sino que, por el contrario, algunos Estados de Europa occidental sostuvieron una elevación de los estándares para igualarlos a lo de los países industrializados y denunciaron las presiones encaminadas a limitar los poderes o restringirlas. MURRAY 2001: 62 .

26 «Considerando que si cualquier nación no adoptare un régimen de trabajo realmente humano, esta omisión constituiría un obstáculo a los esfuerzos de otras naciones que deseen mejorar la suerte de los trabajadores en sus propios países». Considerando de la Constitución de la OIT (de 1919).

$27 \mathrm{Y}$, como hemos apuntado, estrechamente vinculada con la fijación de un estándar de protección, con un alcance más amplio, que permitiese la ampliación efectiva del número de Estados. CheBALi 1989: 204. 
Estados parte de la organización, pasando los países en desarrollo a ser una mayoría ${ }^{28}$.

La ampliación de los Estados parte de la OIT y la decisión de disminuir el nivel de protección para adecuarse a la necesidad de incluir un nuevo mínimo común de protección derivado de la inclusión de países en desarrollo con una legislación laboral mucho menos avanzada, y combinarlo, para evitar la caída de los derechos laborales de aquellos Estados más avanzados, con la cláusula de mayor protección, tuvo, en realidad, una causa mayor: la construcción de la Organización de Naciones Unidas (ONU).

Los mimbres de la construcción de la heredera de la Sociedad de Naciones se fraguaron en la otra costa de los EEUU al pasarse, un año más tarde, de la Convención de Filadelfia (Pensilvania) a California, concretamente, a la ciudad que encarnaba, y lo sigue haciendo, el ideal del progresismo estadounidense: San Francisco. La capital del Bay Area, donde se firmó, en 1945, la Carta de Naciones Unidas. Ya en Filadelfia se había buscado una fórmula que posibilitase la futura incorporación de la OIT dentro de la estructura de la ONU. Esta tuvo lugar poco menos de un año después de la Convención de San Francisco ${ }^{29}$. El 30 de mayo de 1946 la OIT se convirtió en la primera agencia especializada en virtud del art. 57 de la Carta de Naciones Unidas ${ }^{30}$.

28 «El estadounidense David Morse fue Director General entre 1948 y 1970, período durante el cual se duplicó el número de países miembros. La Organización asumió su carácter universal, los países industrializados pasaron a ser una minoría ante los países en desarrollo, el presupuesto creció cinco veces y el número de funcionarios se cuadruplicó»». Información de la web ILO, http://www.ilo.org/global/about-the-ilo/history/lang--es/index.htm (última visita el 28 de marzo de 2017).

${ }^{29}$ La propuesta del Reino Unido de buscar un encaje a la OIT dentro de la OIT y el, todavía, embrión de la ONU, tras una primera oposición de los EEUU, China y Rusia, fue aceptada sin votos en contra. "On 15 May the British delegation moved an amendment to the Dumbarton Oaks proposals to the effect that the ILO should be 'brought into special relationship' with the proposed United Nations and 'should be an important instrument through which should be pursued the object of securing for all improved labour standards, economic advancement and social security'. [...] The amendment was opposed by the Americans, Chinese and Russians. [...] On 2 June the British representative reminded the Committee that his country's proposal was designed to provide formally in the Charter that the ILO should be brought into relationship with the UN and be recognised as one of the principal agencies for pursuing the objectives of Article V of the Atlantic Charter. [...] The Committee accepted the British proposal by 24 votes to 0 [...] that it would be necessary to alter the Constitution of the ILO in order to provide the necessary links with the Organisation». ALCOCK 1971.

30 «They succeeded in slowing down the establishment of links with the United Nations, but not in preventing it, and on 30 May 1946, less than a year after the San Francisco Conference, the Organisation signed an agreement with the United Nations which made it the first of the specialised agencies under article 57 of the United Nations Charter». Chebali 1989: 24. 
Es cierto que la cláusula de la mayor protección de los Derechos Humanos recogida en la Constitución de la OIT se refiere un ámbito sectorial. No cabe duda, por tanto, de su consideración como Derecho Humano evidenciado en la previsión del art. 23 de la Declaración Universal de Derechos Humanos $^{31}$ pero también que su ámbito de aplicación se circunscribe a este ámbito concreto ${ }^{32}$. Por lo tanto, para hablar de la existencia de un principio de derecho internacional de los Derechos Humanos, que supere la consideración de principio sectorial en la aplicación de los estándares de la OIT ${ }^{33}$, es necesario que este constituya una «razón básica» en la construcción del derecho internacional de los Derechos Humanos. Por ello, la consideración de esta cláusula en un ámbito sectorial no la convertiría en un principio general sino que, por el contrario, es necesario que su uso se vea ampliado sustancialmente, como efectivamente ha ocurrido.

\section{CONCLUSIONES}

Entender la lógica de la cláusula de la mayor protección de los DDHH requiere ahondar tanto en su origen como en su naturaleza dentro del derecho internacional de los DDHH. Esto es lo que, precisamente, hemos intentado hacer en el presente artículo. En cambio, este trabajo no agota la riqueza una línea investigadora que requiere un análisis en profundidad de

«The agreement reached was the first between the UN and a Specialised Agency. It contained twenty Articles, and under the first of these the United Nations recognised the ILO as a Specialised Agency responsible for taking such action as may be appropriate under its basic instrument for the accomplishment of the purposes set forth therein». ALCOCK 1971: 202.

31 Art. 23: (1) Toda persona tiene derecho al trabajo, a la libre elección de su trabajo, a condiciones equitativas y satisfactorias de trabajo y a la protección contra el desempleo. (2) Toda persona tiene derecho, sin discriminación alguna, a igual salario por trabajo igual. (3) Toda persona que trabaja tiene derecho a una remuneración equitativa y satisfactoria, que le asegure, así como a su familia, una existencia conforme a la dignidad humana y que será completada, en caso necesario, por cualesquiera otros medios de protección social. (4) Toda persona tiene derecho a fundar sindicatos y a sindicarse para la defensa de sus intereses.

${ }^{32}$ It contains a most favoured protection clause to the effect that the ILO Conventions and resolutions «shall be deemed [not] to affect any law, award, custom or agreement which ensures more favourable conditions to be workers». But only and explicity indicating the workers as beneficiaries, that clause aptly avoids many of the problems pointed out above. Alkema 1998: 53.

33 «with respect to the principle according to which no ILO standard may affect national law or regulations providing more favourable treatment for the workers concerned». Chebali 1989: 23. 
las especificidades de la cláusula en los principales instrumentos internacionales de protección de los $\mathrm{DDHH}^{34}$.

La naturaleza de la cláusula de la mayor protección en el derecho internacional de los DDHH es la de una previsión limitativa extrínseca, esto es, limitadora de una aplicación o interpretación de un derecho reconocido en un tratado internacional que conllevase la limitación de otro instrumento externo al propio tratado.

Una vez delimitada la naturaleza jurídica de la cláusula de la mayor protección y realizada una distinción de aquellas otras cláusulas que impiden una limitación intrínseca, como es el caso de las que tratan de prevenir el abuso de derecho, hemos abordado su origen a través de una reforma de la Constitución de la OIT. La conclusión principal deviene de la lógica de la cláusula y la necesidad de dar respuesta a una problemática planteada ante la inminente extensión de los Estados parte la OIT debido a su incorporación como agencia especializada a una incipiente Organización de Naciones Unidas (ONU). En definitiva, no solo era necesaria una limitación del contenido del tratado para que los Estados en desarrollo pudieran alcanzar, no sin realizar un esfuerzo reformando su derecho interno, el nuevo estándar de protección fijado sino que el éxito de la ampliación pasaba, también, por evitar una consecuencia no deseada, que la protección interna disminuyese en los Estados industrializados.

La reforma de la Constitución de la OIT trató de conjugar esta dicotomía lo que dio paso a su incorporación a la mayoría de tratados internacionales de protección de los DDHH, tanto universales como regionales.

\section{BIBLIOGRAFÍA}

AlCock, A. History of the International Labor Organization, Octagon Books, (New York, 1971).

Alkema, E. A., "The enigmatic no-pretext clause: Article 60 of the European Convention on Human Rights» en Klabbers, Jan y Lefeber, René [ed.] Essays on the Law of Treaties (A collection of essays in honour of Bert Vierdag), Martinus Nijhoof Publishers, The Hague, (Boston, London, 1998): 41-56.

Cabaleiro, E., Los tratados internacionales, la importancia de la nacionalidad de sus destinatarios, Ediciones Rialp, S. A., (Madrid, 1962).

Chebali, V.-Y., The International Labour Organization. A case Study on the Evolution of U.N. Specialised Agencies, Martinus Nijhoff Publishers, Dordrecht, (Boston, London, 1989).

Corten, O. y Klein, P. [ed.], The Vienna Conventions on the Law of Treaties. A commentary, Oxford University Press. (2011): 764 y ss.

${ }^{34}$ Este análisis es abordado en nuestra tesis doctoral que será defendida en los próximos meses. 
Detrick, S., A Commentary on the United Nations Convention on the Rights of the Child, Martinus Nijhoff Publishers, The Hague, (Boston, London, 1999).

Goodman, R. y JinKS, D., «How to influence states: socialization and international haman rights law», Volume 54, Number 3, (2004).

MurRAY, J., «Transnational Labour regulations: The ILO and EC compared», International Studies in Human Rights, Kluwer law international, The Hague, (Boston/London, 2001).

RachovitsA, A., «Treaty Clauses and Fragmentation of International Law: Applying the More Favourable Protection Clause in Human Rights Treaties» en Human Rights Law Review, 16, (2016): 77-101.

TORRES ZúÑIGA, N., «Justiciabilidad de las medidas regresivas de los derechos sociales. Algunas reflexiones acerca de su protección en América Latina» en Derecho PUCP, $\mathrm{n}^{\circ}$ 75, (2015): 95-117.

Vanneste, F., General International Law before Human Rights Courts. Assessing the Specialty Claims of International Human Rights Law, Intersentia, AntwerpOxford-Portland, (2010).

Villinger, M. E., Commentary on the 1969 Vienna Convention on the Law of the Treaties, Martin Nijhoff Publishers, (Leiden/Boston, 2009). 